\section{$\leqslant$ Research Square}

Preprints are preliminary reports that have not undergone peer review.

They should not be considered conclusive, used to inform clinical practice, or referenced by the media as validated information.

\title{
Postsurgical recurrence and metastasis of rectal cancer with T1N0M0 stage
}

\section{Min Wang}

The Second Hospital of Jilin University

\section{Lanqing Cao}

The Second Hospital of Jilin University

\section{Hongyu He}

The Second Hospital of Jilin University

\section{Shuang Wang}

The Second Hospital of Jilin University

Jiannan Li ( $\sim$ jnli@ciac.ac.cn )

The Second Hospital of Jilin University

\section{Case report}

Keywords: CRC, surgery, recurrence, metastasis

Posted Date: February 10th, 2020

DOI: https://doi.org/10.21203/rs.2.23001/v1

License: (c) (1) This work is licensed under a Creative Commons Attribution 4.0 International License. Read Full License 


\section{Abstract}

Background: Post-surgical recurrence and metastasis of colorectal cancer (CRC) with T1NOMO pathological stage hardly occur.

Case presentation: We reported a rare case of post-surgical recurrence and distant metastasis of CRC with T1N0M0 pathological stage. Before the first surgery, electric coloscopy and following pathology examination suspected the rectal mass as adenocarcinoma. The patient underwent radical surgery of the rectal tumor. Postsurgical pathology confirmed the diagnosis of rectal adenocarcinoma with the stage of T1N0M0. At about 3.5 years post the first surgery, the chest and abdominal computed tomography (CT) indicated multiple metastasis in both lungs, a small hepatic cyst, gallstones, flaky shadows in the left 10th rib, the thickening of the intestinal wall of sigmoid colon, pelvic fluid, and bone destruction in the sacral area. The pathology confirmed the recurrence of the rectal adenocarcinoma. As a result, sigmoidectomy, recrectomy, and sigmoid colostomy (Hartmman) were performed. Tumor recurrence and metastasis occurred in this case and Hartmann surgery was performed to improve the life quality of the patient.

Conclusion: Although, most of the CRC patients with T1NOMO pathological stage can recover well after radical tumor resection, timely reexamination and specific diagnosis and treatment plan should still be noted.

\section{Background}

Colorectal cancer (CRC) is a kind of worldwide disease and the incidence rate ranks the third among the malignant tumors [1]. There will be almost 2.2 million new patients diagnosed with CRC per year and 1.1 million deaths since 2030 [2]. Once the patients diagnosed with CRC, surgical treatment is vital to cure the patients or control the rapid progress of the tumor. Further chemotherapy or radiotherapy is always implicated to prevent the recurrence and metastasis of CRC after surgery [3, 4]. According to the National Comprehensive Cancer Network (NCCN) guidelines, radical surgery treatment is necessary and vital for patients with T1N0M0 CRC [5]. But the adjuvant treatment, such as chemotherapy or radiotherapy is not recommended or needed for these patients [5]. Indeed, based on our medical experience and the studies reported worldwide, the post-surgical recurrence and metastasis of CRC with T1N0M0 pathological stage hardly occur.

In the present study, we reported a rare case of CRC with T1N0M0 pathological stage who was observed with post-surgical tumor recurrence and distant metastasis.

\section{Case Presentation}

This study was approved by the Ethics Committee and institutional Review Board of the Second Hospital of Jilin University, Changchun, China. The patient provided informed consent for publication of the case. 
A 58 years old male came to our department with the change of defecation habit for 5 years in January 2016. He complained of loose and liquid stools for more than three times per day. He denied any history of abdominal pain or hematochezia. In addition, the patient also denied a history of hypertension, coronary heart disease, diabetes, acute and chronic infectious diseases, or surgery. The digital rectum examination (lithotomy position) indicated a small mass which was touched with poor activity at 6 o'clock orientation and $6 \mathrm{~cm}$ from the anal edge. No other positive findings were observed through the physical examination. The levels of carcinoembryonic antigen (CEA) and carbohydrate antigen 199 (CA199) were $422.37 \mathrm{ng} / \mathrm{ml}$ and $156.10 \mathrm{U} / \mathrm{mL}$, respectively, which were much higher than the normal levels (CEA, $<3.0 \mathrm{ng} / \mathrm{ml} ; \mathrm{CA} 199,0-35 \mathrm{U} / \mathrm{mL}$ ). Abdominal computed tomography (CT) and pelvic magnetic resonance imaging (MRI) showed a small cyst in the right lobe of liver, gallstones, and the thickening of the rectal wall (Fig. 1). The electric coloscopy showed chronic colitis and colorectal polyps. The "polyp" which was about $6 \mathrm{~cm}$ from the anal edge were under pathological examination. Heterotype glands were found in the tissue for pathological examination and adenocarcinoma could not be excluded. Laparoscopic radical resection of rectal cancer was performed for the patient. The tumor and nearby rectum tissue were resected and the broken ends of the rectum and sigmoid colon were anastomosed. Postsurgical pathology indicated that the tumor was moderately differentiated adenocarcinoma which infiltrated to the submucosa layer (Fig. 2). No cancer tissue was found in the cutting edge and anastomosis area. No tumor metastasis was observed in the lymph nodes (9 lymph nodes were submitted for pathological examination) surrounding the rectum or in the fatty tissues around the root of the inferior mesenteric artery. The rectal cancer was graded as T1NOMO pathological stage. The patient recovered well and discharged from our department.

The patient came to our department 6 months post-surgery for reexamination. Physical examinations and electric coloscopy didn't show any positive findings. The laboratory tests indicated slightly higher level of CEA $(21 \mathrm{ng} / \mathrm{ml})$. The chest and abdominal CT didn't reveal any positive findings, except the hepatic cyst and gallstones.

In July 2019, this patient came to our department again with abdominal distention and vomiting for about 1 week. The stop of defecation also occurred for about 3 days. Slightly abdominal muscle tension was found through physical examination. Much higher levels of CEA (828.57 ng/ml) and CA199 $(349.40 \mathrm{U} / \mathrm{ml})$ were noted which might represent the presence of $\mathrm{CRC}$. The abdominal X-ray showed the gas accumulation and liquid gas level in the intestine (Fig. 3A). The barium enema examination showed intestinal stenosis in the upper rectum that was adjacent to the anastomotic stoma and in the sigmoid colon (Fig. 3B). Chest and abdominal CT indicated multiple tumor metastasis in both lungs, a small hepatic cyst, gallstones, flaky shadows in the left 10th rib, the thickening of the intestinal wall of sigmoid colon, pelvic fluid, and bone destruction in the sacral area (Fig. 4). In addition, the closed lines with high signal were observed in the rectum (Fig. 4). This patient didn't perform electric coloscopy because of the difficulty of the intestinal tract clean.

Finally, this patient underwent exploratory laparotomy. During the surgery, obvious intestinal adhesion was found and the tumor tissues which infiltrated to the sacral bones were also noted. As a result, 
sigmoidectomy, recrectomy, and sigmoid colostomy (Hartmman) were performed. Pathological examination confirmed the recurrence of rectal adenocarcinoma (Fig. 5). This patient discharged from our department at 10 days post-surgery.

\section{Discussion}

According to the NCCN guidelines, radical resection is enough for the treatment of rectal cancer with T1N0M0 pathological stage [5]. There is no need to perform the adjuvant therapy for these patients [6]. In our study, this patient underwent radical resection of the rectal tumor and the histopathological examination indicated that the tumor tissues infiltrated to the submucosa layer. As a result, the cancer was graded as T1N0M0 pathological stage. Based on NCCN guidelines, chemotherapy or radiotherapy was not performed for this patient. Following reexamination was only performed in the 6th month postsurgery and no signs of tumor recurrence and metastasis were found. However, at about 3.5 years postsurgery, this patient presented with serious intestinal obstruction which was a result of tumor recurrence and intestinal adhesion. Higher CEA and CA199 levels might also reveal the presence of gastrointestinal cancer. The chest and abdominal CT indicated the tumor recurrence which invaded the sacral area and the tumor metastasis in both lungs and maybe in the 10th rib. Pathological examination further confirmed the recurrence of rectal cancer.

As far as we know, the post-surgical recurrence and metastasis of rectal cancer with T1N0M0 pathological stage hardly happen. In addition, for these patients, there is no need to perform the adjuvant therapy post-surgery. However, in our case, tumor recurrence and metastasis indeed occurred in T1N0M0 staged CRC patient. The post-surgical pathological examination confirmed the radical resection of the tumor in this case. A study conducted by Christina Curtis et al. confirmed that systemic spread can occur early in CRC [7]. This is different from our previous cognition that tumor recurrence and metastasis occur in the advanced stage. However, little is known about the time of tumor metastasis or the molecular mechanism which can control the tumor metastasis [8, 9].

Even though, NCCN guidelines is vital for the treatment of CRC. Post-surgical reexamination is important for these patients. In our case, the patient didn't perform the reexamination at the pre-set intervals. Therefore, the tumor recurrence and metastasis weren't noted in time. In recent time he came to our hospital, the tumor recurrence and metastasis are so obvious that little could be done to improve his overall survival time. In addition, specific treatment plan, such as gene therapy and immunity therapy, is recommended for $\mathrm{CRC}$ patients, including those with early pathological stage.

\section{Conclusion}

In the present study, we reported a rare case of postsurgical recurrence and metastasis of rectal cancer patient with T1N0M0 stage. Although, most of the CRC patients with T1N0M0 or T2N0M0 pathological stage can recover well after radical tumor resection, timely reexamination and specific diagnosis and treatment plan should still be noted. 


\section{Declarations}

\section{Acknowledgement}

Not applicable.

\section{Authors' contributions}

JNL and SW designed the report. LQC performed the pathological analysis. HYH and MW collected the patient's clinical data and information. MW and JNL wrote the paper. All authors read and approved the final manuscript

\section{Funding}

None.

\section{Availability of data and materials}

All data generated or analyzed are included in this published article.

\section{Ethics approval and consent to participate}

Not applicable.

\section{Consent for publication}

Consent for publication was obtained from the patient described in this article.

\section{Competing interests}

The authors declare that they have no competing interests.

\section{Compliance with ethics guidelines}

Min Wang, Lanqing Cao, Hongyu He, Shuang Wang, and Jiannan Li declare no conflicts of interest. This manuscript is a case report article and does not involve a research protocol requiring approval by the relevant institutional review board or ethics committee.

\section{References}

[1] M. Lorans, E. Dow, F.A. Macrae, I.M. Winship, D.D. Buchanan, Update on Hereditary Colorectal Cancer: Improving the Clinical Utility of Multigene Panel Testing, Clinical Colorectal Cancer 17(2) (2018) E293E305.

[2] M. Arnold, M.S. Sierra, M. Laversanne, I. Soerjomataram, A. Jemal, F. Bray, Global patterns and trends in colorectal cancer incidence and mortality, Gut 66(4) (2017) 683-691. 
[3] S. Rosello, F. Papaccio, D. Roda, N. Tarazona, A. Cervantes, The role of chemotherapy in localized and locally advanced rectal cancer: A systematic revision, Cancer Treatment Reviews 63 (2018) 156-171.

[4] T. Watanabe, K. Muro, Y. Ajioka, Y. Hashiguchi, Y. Ito, Y. Saito, et al., Japanese Society for Cancer of the Colon and Rectum (JSCCR) guidelines 2016 for the treatment of colorectal cancer, International Journal of Clinical Oncology 23(1) (2018) 1-34.

[5] A.B. Benson, III, A.P. Venook, M.M. Al-Hawary, L. Cederquist, Y.-J. Chen, K.K. Ciombor, et al., NCCN Guidelines (R) Insights Colon Cancer, Version 2.2018 Featured Updates to the NCCN Guidelines, Journal of the National Comprehensive Cancer Network 16(4) (2018) 359-369.

[6] T. Shinagawa, T. Tanaka, H. Nozawa, S. Emoto, K. Murono, M. Kaneko, et al., Comparison of the guidelines for colorectal cancer in Japan, the USA and Europe, Annals of Gastroenterological Surgery 2(1) (2018) 6-12.

[7] Z. Hu, J. Ding, Z. Ma, R. Sun, J.A. Seoane, J.S. Shaffer, et al., Quantitative evidence for early metastatic seeding in colorectal cancer, Nature Genetics 51(7) (2019) 1113-+.

[8] S. Turajlic, C. Swanton, Metastasis as an evolutionary process, Science 352(6282) (2016) 169-175.

[9] A.W. Lambert, D.R. Pattabiraman, R.A. Weinberg, Emerging Biological Principles of Metastasis, Cell 168(4) (2017) 670-691.

\section{Figures}



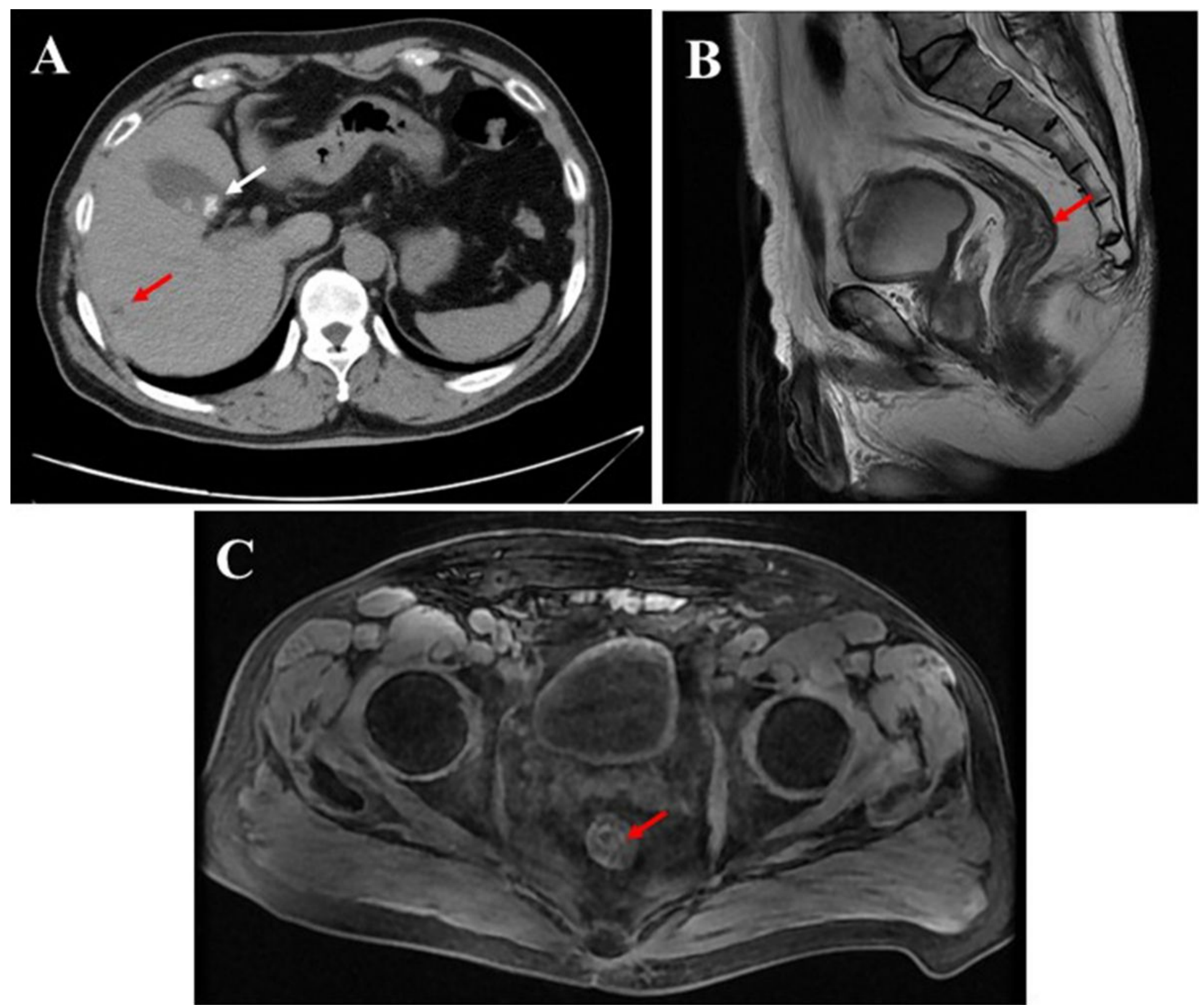

Figure 1

$\mathrm{CT}$ and MRI findings pre-surgery. (A) Abdominal CT showed a small hepatic cyst (red arrow) and gallstones (white arrow). (B, C) Pelvic MRI indicated the thickening of rectal wall (red arrows). 


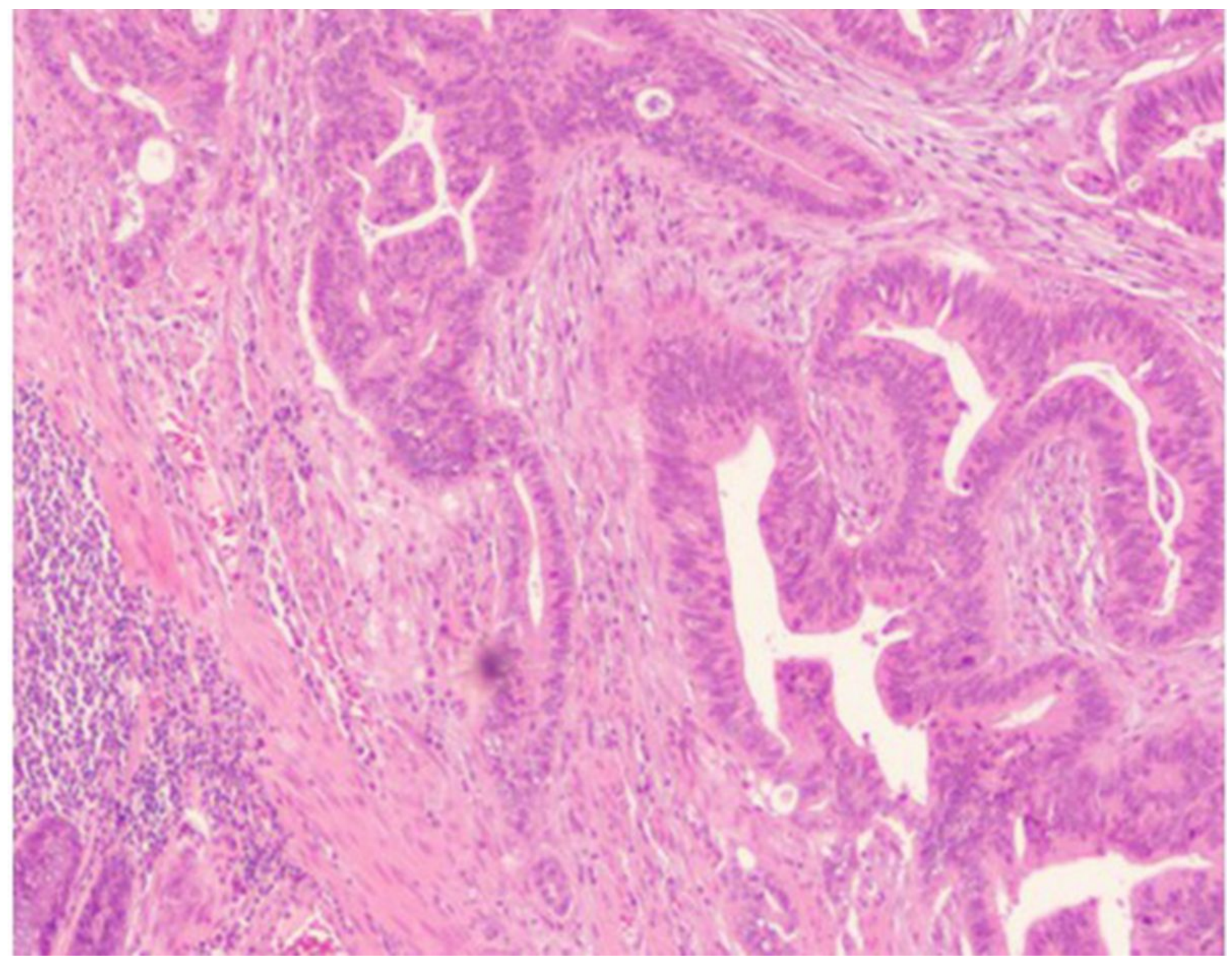

\section{Figure 2}

H\&E staining of rectal cancer (malignant $\times 40$ ). 

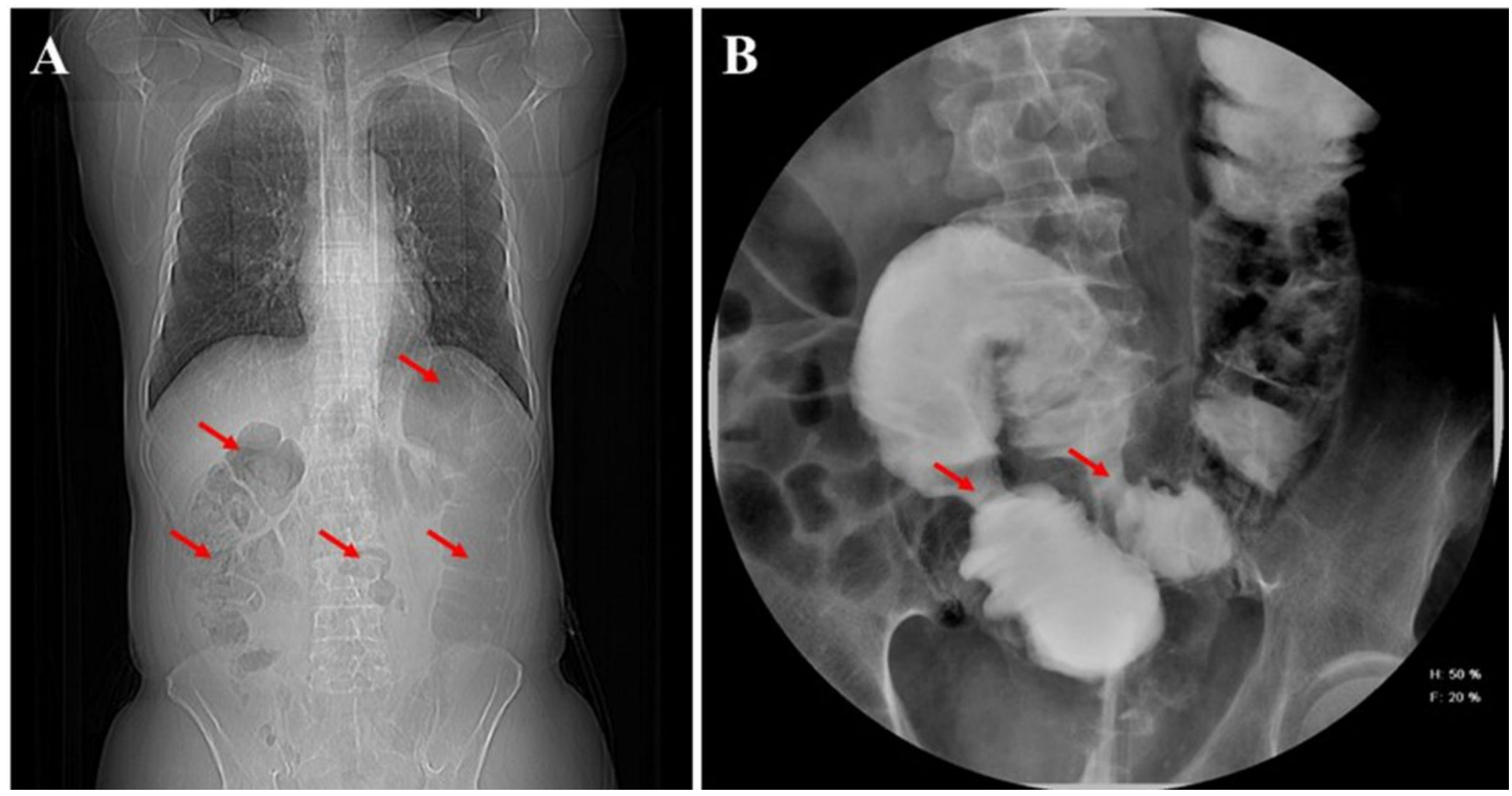

Figure 3

Abdominal X-ray and barium enema examination confirmed the diagnosis of intestinal obstruction. (A) Abdominal X-ray showed gas accumulation and liquid gas level (red arrows). (B) The barium enema examination showed intestinal stenosis (red arrows).
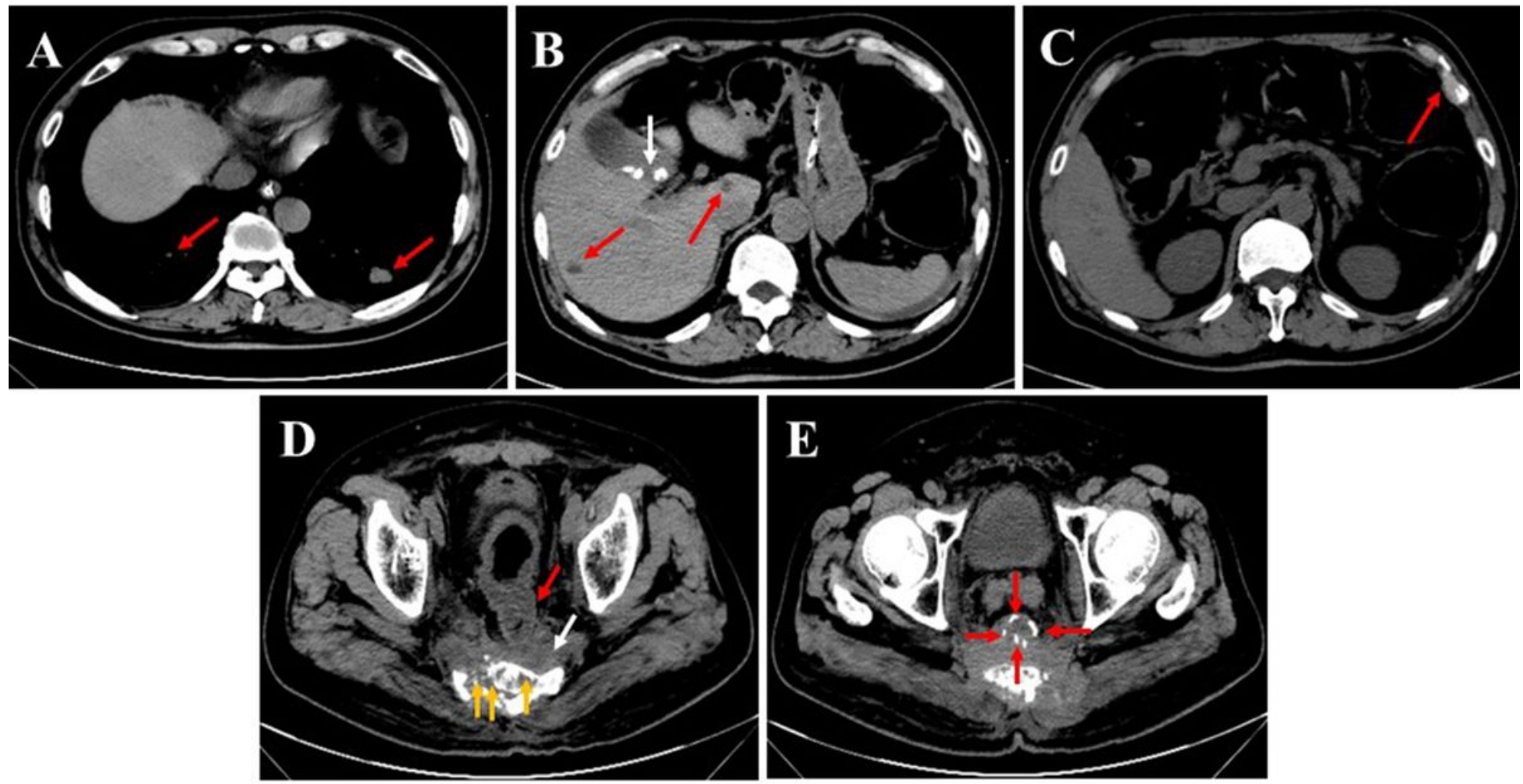

Figure 4 
Chest and abdominal CT exanimation. (A) Tumor metastasis (red arrows) in both lungs. (B) Hepatic cysts (red arrows) and gallstones (white arrow). (C) Flaky shadows in the left 10th rib. (D) The thickening of the wall of sigmoid colon (red arrow), pelvic fluid (white arrow), and bone destruction in the sacral area (yellow arrows). (E) The closed lines with high signal in the rectum (red arrows).

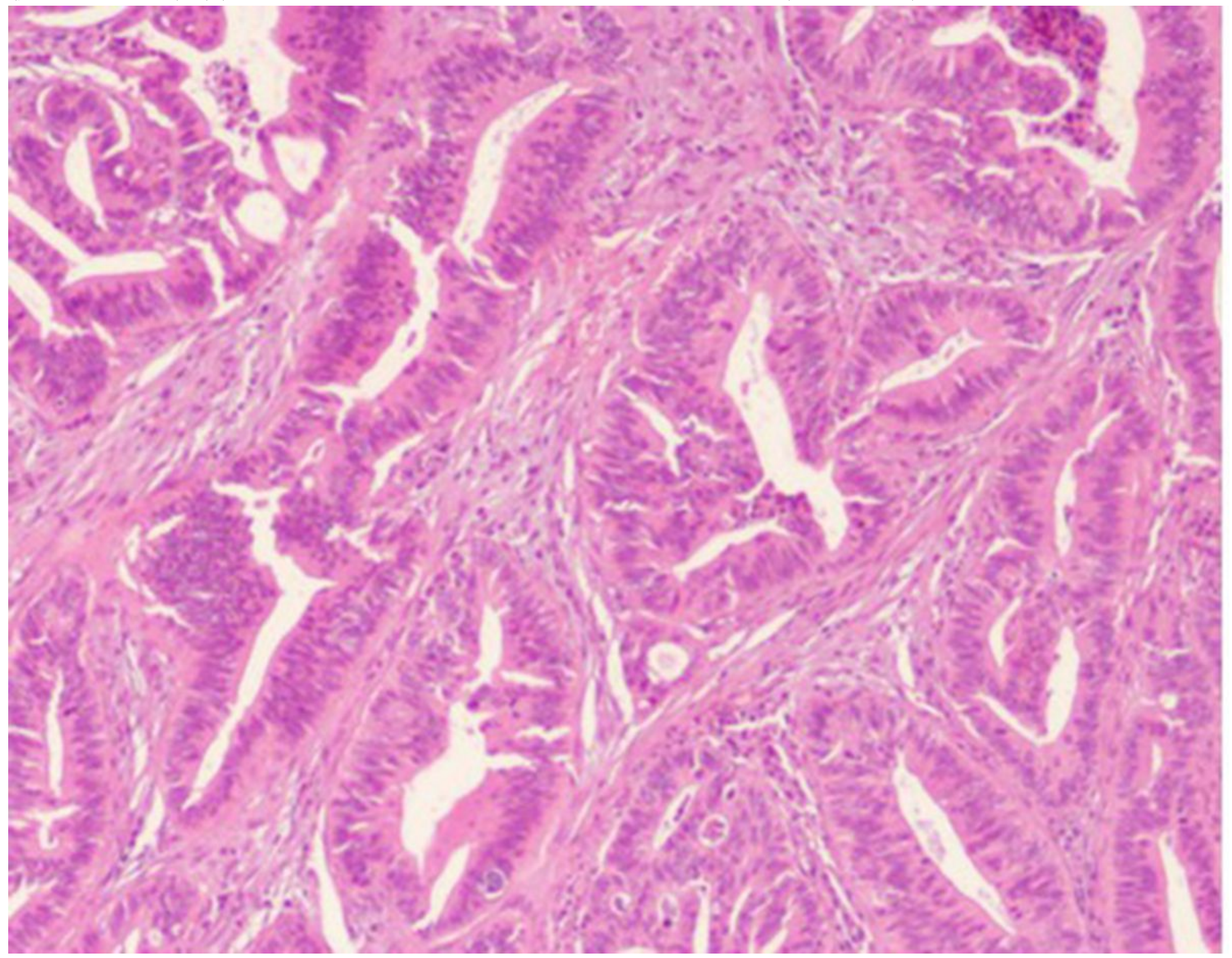

\section{Figure 5}

H\&E staining confirmed the CRC recurrence (malignant $\times 40$ ). 